\title{
Optimizing sterilization logistics in hospitals
}

\author{
Joris van de Klundert • Philippe Muls • \\ Maarten Schadd
}

Received: 5 December 2006 / Accepted: 12 July 2007 / Published online: 25 September 2007

(C) Springer Science + Business Media, LLC 2007

\begin{abstract}
This paper deals with the optimization of the flow of sterile instruments in hospitals which takes place between the sterilization department and the operating theatre. This topic is especially of interest in view of the current attempts of hospitals to cut cost by outsourcing sterilization tasks. Oftentimes, outsourcing implies placing the sterilization unit at a larger distance, hence introducing a longer logistic loop, which may result in lower instrument availability, and higher cost. This paper discusses the optimization problems that have to be solved when redesigning processes so as to improve material availability and reduce cost. We consider changing the logistic management principles, use of visibility information, and optimizing the composition of the nets of sterile materials.
\end{abstract}

Keywords Sterilized logistics • Logistic design • Outsourcing • Optimization • Complexity

\section{Introduction}

The cost of health care in developed countries is around $10 \%$ [2] of gross domestic product and is expected to increase. Hence, health care cost places an increasingly heavy burden on national and individual budgets, and many developed countries are implementing policies to (stimulate health care providers to) cut cost.

J. van de Klundert $(\varangle) \cdot$ P. Muls · M. Schadd

Department of Quantitative Economics,

Faculty of Economics and Business Administration,

Universiteit Maastricht, PO Box 6166200 MD,

Maastricht, The Netherlands

e-mail: jj.vandeklundert@ke.unimaas.nl
There is a widespread belief that the cost effectiveness of health care can be improved. Carter [2] claims that a cost cut in health care by 10 to $20 \%$ is possible. For hospitals in The Netherlands, a recent report [1], written on behalf of the secretary of health care, identifies opportunities for improvement in logistics of goods and pharmaceuticals of 1 billion euros, and another 2 billion euros in patient logistics. This report also concludes that a $20 \%$ cost reduction is possible. The larger part of the cost reduction can be achieved by relatively straightforward measures such as adopting uniform work processes, standardizing materials, quantity discounts, et cetera. In addition, better planning, and appropriate use of information technology are identified as valuable improvement opportunities.

In this paper, we explore opportunities for improvements in a specific flow of goods in hospitals, i.e., the flow of sterile instruments. The improvements are based on an aligned combination of improvements in work processes and information technology.

A typical Dutch hospital-which is not different from hospitals in other developed countries-has invested millions of euros in sterile instruments. On a national level, the investment in sterile equipment can be estimated to exceed 500 million euros. Moreover, central sterilization service departments (CSSD's), are capital intensive and, at a national level, employ thousands of people. Whether it is via taxes or via insurance payments, the expenses that come with the required availability of sterile instruments are paid by the customer, the patient.

The sterile logistics processes also incur a high opportunity cost. In many hospitals, the CSSD is located near the operating theatre (OT), in a central position in the hospital. Using the valuable space near the OT for 
care and cure rather than for the secondary sterilization processes provides opportunities to improve the service to the customers.

The optimization of the logistics of sterilized items in hospitals has received little attention in scientific literature. The paper by Fineman and Kapadia [3] deals with establishing appropriate stock levels, under the assumption of constant demand. Although their model is different, the issue is closely related to the topics presented in this paper. Fineman and Kapadia [3] consider partitioning the stock into two components. The first component is the processing stock which is kept because of the replenishment cost. The second component consists of replacement stock, the stock for unanticipated use, which in their view can be due to instrument wear, instruments being lost or damaged, et cetera.

To the best of our knowledge, there are no later works on optimization of sterile logistics in the scientific literature. Perhaps, the lack of scientific interest must be explained by the lack of priority given to controlling the cost of the secondary process of sterile logistics by hospitals themselves. Instead the focus has been on its reliability. If contaminated instruments are not cleaned and sterilized well, they may cause serious infections to other patients. Similarly, if poor logistics management leads to instruments being lacking when needed, it may endanger patient lives.

As mentioned before, hospitals are currently under pressure to become more effective. Moreover, it is widely recognized that cost reductions in secondary processes free money to improve the primary processes of patient cure and care. Consequently, attention for optimizing the logistic processes involved in sterile logistics, centered around the CSSD and the OT, has increased. In particular, there is much attention for outsourcing of the CSSD. Examples of such initiatives can be found in The Netherlands, and Belgium [5]. In whatever the form is, placing the CSSD at a distance, or even changing the logistic principles of the sterile logistics, entails the risks of lowering sterile item availability and increasing cost, rather than reducing them. Whether the cost effectiveness will improve, depends on the extent to which the logistic design and operation are optimized. This paper deals with these optimization problems.

Before we start the analysis however, let us briefly elaborate on the current information technology situation. Of course, the demand for sterile instruments in the OT is determined by the surgeries taking place. Some of these surgeries are planned, others may be emergencies. Most hospitals enter information on planned operations into the Hospital Information System (HIS). However, it is not uncommon for CSSD activities to be executed and planned without making use of HIS information. Moreover, in many hospitals, the HIS does not provide information on the whereabouts of sterile instruments during the day, and hence cannot take it into account when planning and executing the sterilization activities. At present, RFID technology promises to make real time information on instruments available, but some difficulties remain to be solved. Moreover, hardware alone will not solve the problem. Software and organization, as needed to make effective use of relevant HIS functionality, are challenges as well.

The logistical and mathematical concepts which are investigated in this paper stem from joint projects with the Maasland Ziekenhuis in Sittard, The Netherlands. In the process of building a new customer centered hospital, and preparing new working processes, it is realizing improvements which are based on the same concepts. Prior to improving logistical concepts it has worked hard on standardization of surgery processes and required instruments, and implementing supporting ICT systems. We will address the practical progress that is made, or about to be made soon, in the final section. In this final section we also reflect on the relevance of the findings.

In Section 2 we start the analysis by modelling the sterile logistics, and considering several logistic principles to manage the flow of sterile goods. Gradually we will move towards a more demand triggered process, and recalculate optimal inventory levels. Section 3 addresses the basic deterministic optimization problems arising in this setting. The resulting cost minimization problems involve transportation and inventory cost. The presented problem formulations are akin to lot sizing and transportation problems, and are shown to be solvable in polynomial time by dynamic programming techniques. Section 4 extends these results to dynamic, nondeterministic, problem settings. In particular it addresses the value of real time information, as e.g. becomes available when using RFID technology. Section 5 deals with the issue of optimally composing nets of sterile items, so as to reduce overall logistics cost. We address the complexity of several problem variations, and show a general version of the problem to be NP-hard. We present an integer linear programming formulation of the problem which appears to be time consuming to solve.

\section{Basic logistic design}

A rudimentary design for sterile logistics and subsequent improvements is presented in [5, 6]. For ease of 
exposition (and since the aforementioned publications are in Dutch), we now briefly repeat the presented design and its improvements (see also Fig. 1). We consider the flow starting from the sterile storage of the operation theatre. Here the sterile instruments are placed in stock. They are not stocked individually here, but grouped in nets. Typically, all items in one net are exactly the items needed for a particular surgery. However in general this need not be the case. It may be the case that the content of a net is more general, so as to apply to several types of surgery, or that one type of surgery requires nets of distinct types.

Shortly before an operation, the required nets are taken from the storage, put onto a cart, and this cart will be taken to the required OT room. During the operation, the sterile items, whether they are used or not, will become contaminated. When the surgery is finished, all materials will be brought to the contaminated storage of the OT, from where they are taken to the goods receipt of the CSSD. There they are dismounted, disinfected, perhaps precleaned, and subsequently put into the washing machines. After washing, the materials are regrouped to form nets. The nets are put into the autoclaves where the sterilization takes place. Once sterilized, the nets are placed in the sterile storage of the CSSD. From there they are brought to the sterile storage of the OT, which completes the closed loop.

Typically, usage plus replenishment takes more than half a day, even when the CSSD is next to the OT. Therefore, we assume throughout this paper that when the CSSD is outsourced, sterile nets can be used only once per day.

Within this closed loop of the sterilization logistics, the point of consumption of the sterile instruments is the OT. The utility of the material is therefore highest when it is in the sterile storage of the OT. An effective logistic control principle is thus to replenish all items to this sterile storage of the OT as quickly as possible. In real life, many hospitals process all instruments that are taken from the sterile storage of the OT through the entire loop in one day, so that at the end of the day, they are again in the sterile storage of the OT. This basic control principle also has several disadvantages. First of all, it requires maximum storage capacity at a place in the hospital, near the OT, where space is most valuable for the primary process. Second, it may involve working extra hours by the CSSD for materials that are not needed the next day. Thirdly, it may incur unnecessary transportation.

We now present our basic model to analyse logistic design of sterile logistics. The model optimizes the process on a weekly basis. The weekly cost have three constituents, namely the transportation cost, the OT storage cost, and the instrument cost. The transportation cost is linear in the number of transports to the OT. The storage cost at the OT is linear in the required OT storage space. For ease of analysis we assume that the storage space of a net is proportional to the number of instruments it contains, but the models allow for other relationships as well. Finally, we consider the
Fig. 1 Rudimentary design for sterile logistics and subsequent improvements

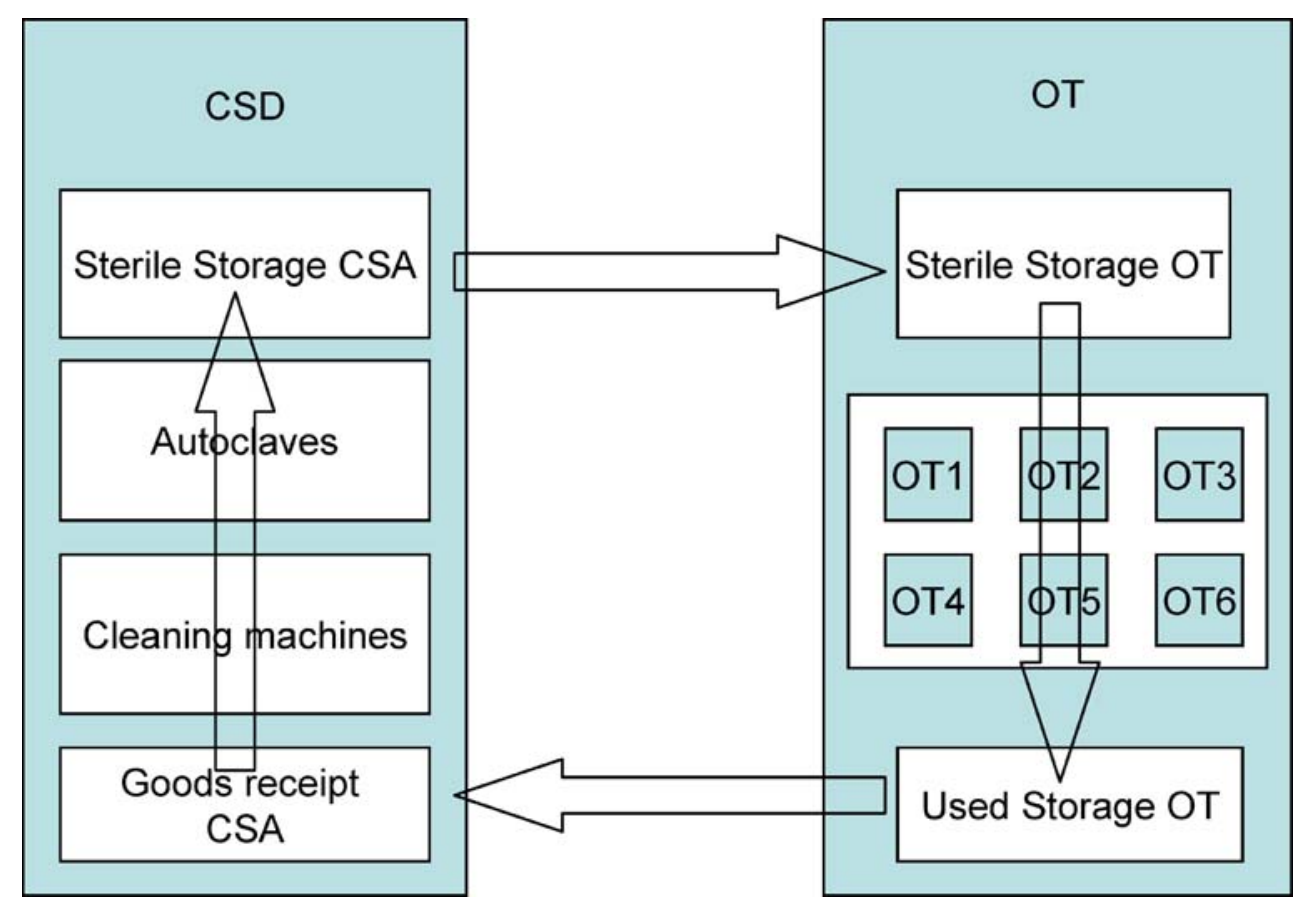


instrument unit cost which models the cost incurred by usage, handling, and sterilization of an instrument. The instrument unit cost is linear in the number of times an instrument is used. These three types of costs are the most important to the hospital when outsourcing the CSSD. It is not hard to see that when outsourcing the CSSD, transportation cost goes up, while at the same time storage cost at the OT might go down. The instrument unit cost will come into play in a later stage.

Example 1 The example below will serve as the running example. In the example there are five different operation types $\{A, B, C, D, E\}$. There are dedicated nets of instruments for each of the operation types. The execution of each of the operations requires instruments of the eight instrument types $\{a, b, c, d, e, f, g, h\}$. The requirements are as follows:

1. Operation A:(a,f,g)

2. Operation B:(b,f,g)

3. Operation C:(c,g)

4. Operation D: $(\mathrm{d}, \mathrm{h})$

5. Operation E:(e,h)

Planned operations take place according to the following weekly schedule:

1. Monday morning: 3A, 6D

2. Monday afternoon: $3 \mathrm{~B}: 6 \mathrm{D}$

3. Tuesday morning: $3 \mathrm{~A}, 6 \mathrm{D}$

4. Tuesday afternoon: 3C: 6D

5. Wednesday morning:: $1 \mathrm{C}, 1 \mathrm{E}$

6. Wednesday afternoon: $1 \mathrm{~B}: 1 \mathrm{E}$

7. Thursday morning: $3 \mathrm{C}, 6 \mathrm{E}$

8. Thursday afternoon: 3B: $6 \mathrm{E}$

The cost parameters are set as follows:

1. Transportation cost (per transport $)=40$;

2. Instrument usage cost (per instrument $)=1$;

3. Storage cost (per capacity unit) $=9$;

The basic logistic design described above requires that all nets are being kept in storage simultaneously at the OT. Since nets can be used only once per day, the schedule implies that 3 nets for each of A, B and C, and 12 nets for each of $\mathrm{D}$ and $\mathrm{E}$; in total 33 nets must be kept in storage simultaneously.

The capacity requirements for the nets are one unit per instrument, yielding a capacity requirement of 3 for nets of type $A$ and $B$, and 2 for the other nets. Thus total storage cost amounts to $((3 \times 3)+(3 \times 3)+(3 \times 2)+$ $(12 \times 2)+(12 \times 2)) \times 9)=(72 * 9)=648$. Transportation is required once per day, for 4 days, yielding transportation cost of $4 \times 40=160$. Finally, the instrument cost amounts to 129 , the number of instruments required to execute the weekly schedule. Total cost are therefore 937. If we assume that the transportation cost of 40 results from outsourcing the CSSD, the setting in which the CSSD is not outsourced has a cost of $937-$ $160=777$. Hence this 777 will serve as the reference cost (see Column 1 of Table 1).

Purposely continuing to neglect unplanned use of sterile nets for the time being, the initial effect of outsourcing the CSSD is an increase in total cost of 160 being the transportation cost. This assumes that the storage cost remains unaltered. In fact, when outsourcing the CSSD, storage cost at the CSSD may increase to keep sterile nets in storage, whereas storage capacity in the hospital may decrease. In subsequent computations, we assume that there is an opportunity cost involved for every unit of storage capacity saved in the hospital. The opportunity costs take cost reductions by using cheaper storage at a remote CSSD into account, but might ultimately even include benefits from alternative use of hospital space. It may be difficult to estimate in real life, but it exists. It is important since its value must counterbalance the increase in transportation cost to make the outsourcing cost effective.

In the next section we explore logistic improvements which utilize the aforementioned possibilities. A first change in logistic design arises when part of the inventory that is currently kept at the sterile storage of the OT is moved to the sterile storage of the CSSD. We start by minimizing total cost, i.e. the sum of the transportation and holding cost. In a separate section, we address non deterministic settings in which part of the surgeries are emergencies instead of planned surgeries.
Table 1 Different solutions and cost comparison

\begin{tabular}{lclcc}
\hline Cost & Push & Pull daily & Pull twice daily & Optimal \\
\hline transportation cost & 0 & 160 & 320 & 280 \\
instrument usage cost & 129 & 129 & 129 & 129 \\
storage cost & 648 & 189 & 0 & 36 \\
total cost & 777 & 478 & 449 & 445 \\
\hline
\end{tabular}




\section{Deterministic optimization}

The models in this section explicitly assume all surgeries and the resulting sterile instrument usage are completely predictable. In this case, sterile storage at the OT is not required. It is possible to deliver sterile items from the CSSD sterile storage just in time before the surgery begins. When transportation cost is high, this Just In Time design may lead to a cost increase. On the other hand, a cost reduction might be attainable when using a small sterile storage at the OT, to reduce the number of required transportations (but with an increase in storage cost).

Notice that in our basic model storage cost at the sterile storage of the OT depends on the capacity of the storage, not on the inventory levels. (Nothing is to be gained from reducing inventory levels at the OT, if it leads to higher inventory levels in another part of the closed loop process.) Consequently, minimization of the transportation cost for a given OT schedule, takes the sterile storage capacity at the OT as an input parameter. We now model the resulting problem mathematically.

As a first modelling step we notice that any reasonable delivery schedule can be assumed without loss of generality to deliver instruments in the order in which their corresponding operations are scheduled. Moreover, the set of moments in time at which delivery occurs in an optimal solution can be discretized: materials are required at the start of an operation, and hence only the starting time moments need to be considered. However, in practice, OT rooms are scheduled in blocks which contain sequences of operations. In this case, the delivery moments in an optimal solution can be assumed to occur at the start of a block.

In combination with the delivery order, this discretization yields that the joint optimization of the transportation cost and the storage cost only needs to consider the volume of the instruments required in each block. To see this, consider the case where a set of delivery moments is given. Then, the instruments delivered at each of these moments can easily be determined: simply deliver all instruments required for the blocks whose starting time falls between the current and the next delivery. Obviously, later delivery yields the solution to be infeasible. Earlier replenishment can only lead to an increase of required storage capacity. Thus, given the delivery moment, it is easy to decide when to deliver each of the required nets. Hence, the problem boils down to selecting a set of delivery moments which minimizes total cost.

Now, let $\left(t_{1}, \ldots, t_{m}\right)$ be the set of delivery opportunities for sterile nets as implied by the OT program.
We let $Q_{i}=1$ if delivery takes place at $t_{i}$ and zero otherwise, for $i=1, \ldots, m$. The cost per delivery is denoted by $d$. The transportation capacity is modeled by $Q$, and $q_{i}$ is the volume delivered at $t_{i}, i=1, \ldots, m$. We denote by $S$ the storage capacity at the OT, by $e$ the storage cost per unit, and by $c_{i}, i=1, \ldots, m$ the volume required for the sterile nets needed at time $t_{i}$. For $i=$ $0, \ldots, m, h_{i}$ denotes the inventory position at time $i$. (Thus, the starting inventory $h_{0}$ is also appropriately defined). Now, the problem can be straightforwardly modelled as:

$$
\begin{aligned}
& \min C=d \times \sum_{i=1}^{m} Q_{i}+e \times S \\
& Q_{i} \times Q \geq q_{i} \quad i=1 \ldots, m \\
& h_{0}=0 \\
& h_{i}=h_{i-1}+q_{i}-c_{i} \quad i=1, \ldots, m \\
& h_{i} \leq S \quad i=0, \ldots, m \\
& h_{i} \geq 0 \quad 0=1, \ldots, m \\
& q_{i} \geq 0 \quad i=1, \ldots, m \\
& Q_{i} \in\{0,1\} \quad i=1, \ldots, m
\end{aligned}
$$

This model assumes that sterile nets which are used in the block immediately after delivery never use storage capacity. Should one prefer to model that these nets do require storage, the storage capacity constraint (4) simply becomes

$h_{i}+c_{i} \leq S \quad i=1, \ldots, m$.

The case in which the capacity of the transportation vehicle is unbounded is a special case which can be modeled by setting $Q$ sufficiently large (e.g by setting $\left.Q=\sum_{i=1}^{m} c_{i}\right)$. This special case corresponds to the real life situation where the transportation capacity is never restricting, as is for instance the case when transportation capacity is adequately arranged in advance.

As is clear from the graph representation in Fig. 2, the resulting problem is a special case of the fixed charge network flow problem, the decision version of which is in general known to be NP-Complete [4]. If the capacity of the transportation device is bounded, and nets have non unit capacity requirements, the decision version of the delivery problem can easily seen to be strongly NP-Complete, since it contains the 3 Partitioning problem (see e.g. [4]) as a special case. In the remainder we consider the case of unbounded transportation capacity. 
Fig. 2 Flow graph

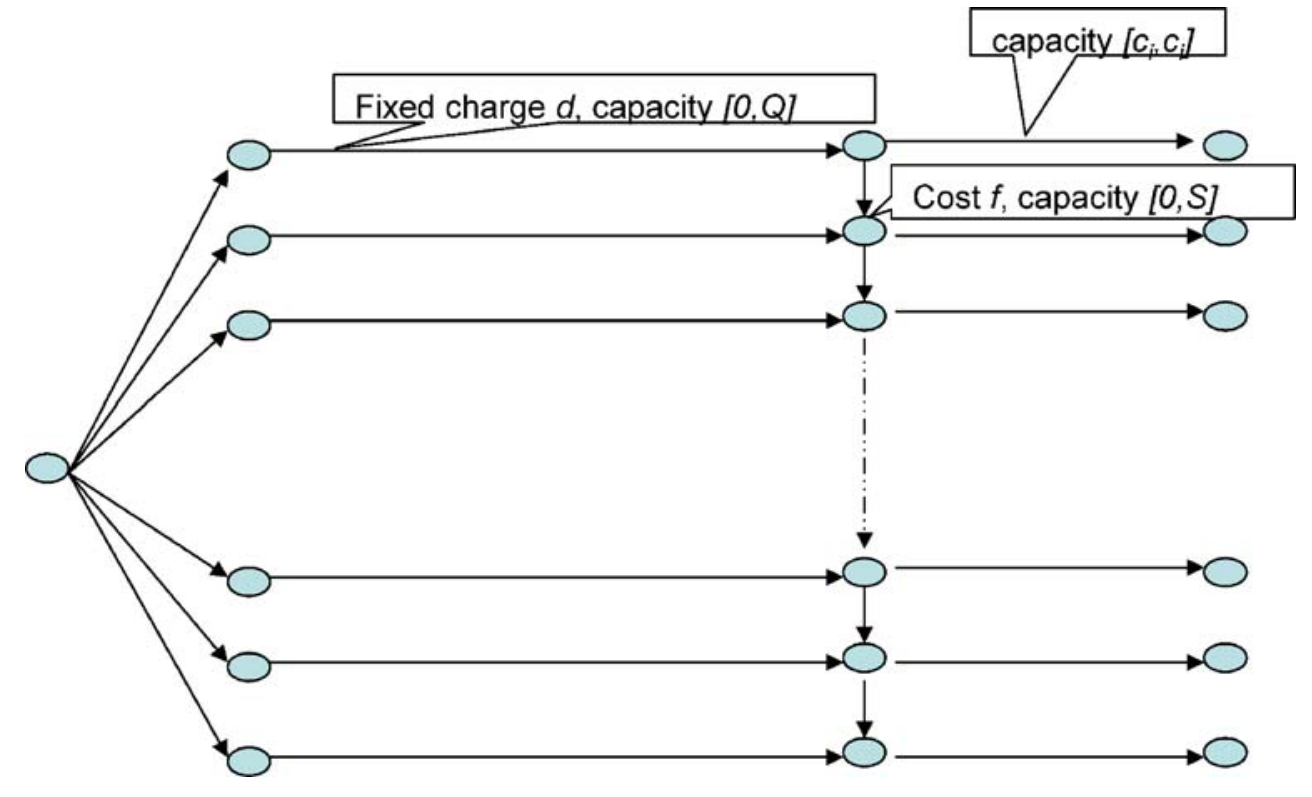

The capacitated version of the problem in which $S$ is fixed can be easily solved. Since storage cost has become fixed at $e \times S$, cost $C$ is minimized by minimizing the number of deliveries. This is achieved by repeatedly delivering a quantity of $S$ at the latest time moments $i$ possible without rendering the solution to become infeasible. Thus, a quantity $S$ is delivered at $t=0$, a second delivery at the earliest time moment $i$ for which $\sum_{k=0}^{i} c_{k}>S$, et cetera. The resulting delivery moments, and hence the resulting transportation cost, can thus be computed in $O(m)$ time. We denote this cost, for given storage capacity $S$, by $C(S)$.

Now let us turn to the strategic problem of determining the optimal storage capacity $S^{*}$, as we are aiming to solve the strategic issue of jointly optimizing transportation cost and storage cost. We let $S^{*}$ be a storage capacity such that

$C\left(S^{*}\right)=\min _{S} C(S)$.

Moreover, for $1 \leq i<i^{\prime} \leq m$ define $c_{i i^{\prime}}=\sum_{k=i}^{i^{\prime}} c_{k}$. Then it is not hard to see that $S^{*}=c_{i i^{\prime}}$ for some $1 \leq i<$ $i^{\prime} \leq m$. Hence the following straightforward procedure solves the problem of determining $S^{*}$ and a set of corresponding delivery moments.

1. For all $1 \leq i<i^{\prime} \leq m \operatorname{set} c_{i i^{\prime}}=\sum_{k=i}^{i^{\prime}} c_{k}$.

2. For all distinct values of $c_{i i^{\prime}}$ compute $C\left(c_{i i^{\prime}}\right)$

3. $C \rightarrow \min _{i, i^{\prime}} C\left(c_{i, i^{\prime}}\right)$.

The time complexity of this procedure is $O\left(\mathrm{~m}^{3}\right)$. The practical relevance of solving this model with variable storage capacity is not to reoptimize storage capacity whenever the OT schedule changes. Instead, it facilitates solving various scenarios to optimality, to develop an understanding of how storage capacity, OT schedules, and transportation schedules interact, and determine total cost.

We now briefly consider a variation where storage cost is replaced by holding cost. In this model the cost of keeping inventory at the OT does not depend on the storage capacity $S$ but are modelled as $f \times h_{i}$ for each time interval between two consecutive delivery moments, $f$ being the per unit holding cost. The cost minimization problem can then be modelled as:

$\min F=d \times \sum_{i=1}^{m} Q_{i}+f \times h_{i}$

$Q_{i} \times Q \geq q_{i} \quad i=1 \ldots, m$

$h_{0}=0$

$h_{i}=h_{i-1}+q_{i}-c_{i} \quad i=1, \ldots, m$

$h_{i} \leq S \quad i=1, \ldots, m$.

$q_{i}, h_{i} \geq 0 \quad i=1, \ldots, m$

$Q_{i} \in\{0,1\} \quad i=1, \ldots, m$ 
Again, we denote by $F(S)$ the value of the optimal solution for this problem for given $S$. The problem of finding $F(S)$ once $S$ is given, can be solved by the following dynamic programming recursion. For every delivery opportunity $t_{i}$ we define $c(i, h)$ to be the minimum cost over all feasible replenishment strategies that result in an inventory level of $h$ at time $t$. We require $h_{0}=0$, and we set $c(0,0)=0$ and $c(0, h)=+\infty$ for $h=1, \ldots, S$. Now, $c(i, h)$ can be defined as follows:

$c(i, h)=\min \left\{c\left(i-1, h+c_{i}\right)+e \times\left(h+c_{i}\right)\right.$,

$\left.d+\min _{q=1}^{Q} c\left(i-1, h-q+c_{i}\right)+e \times\left(h-q+c_{i}\right)\right\}$

Now let $S$ be the maximum stock level considered ( $S$ can be the storage capacity or any other upperbound on $h$ ). Then, the recursion yields a straightforward dynamic programming algorithm of $O(Q \times S \times m)$ time complexity for the problem of finding the optimal replenishment strategy. Notice that this time complexity is pseudopolynomial.

We conclude this session by applying the aforementioned methods to the running example.

Example 2 In the previous solution transportation took place once per day, to completely refill OT inventory. A first and major improvement is realized when delivering every day at the beginning of the day, the nets required for that day. This leads to a reduction in storage cost, while keeping transportation cost unchanged. Notice that since we assume that storage is only needed for nets used in blocks which start later than their delivery, storage is only needed for nets used in the afternoon. This yields a storage cost of $\max (21+18+5+21) * 9=189$. The overview is given in Column 2 of Table 1.

In the example, this strategy of outsourcing the CSSD and implementing a pull strategy for delivery of sterile instruments, yields a significant (40\%) cost reduction. Further cost reduction is possible by optimizing transportation, as can be concluded from Column 3 in Table 1. Column 4 gives the optimal solution, as it results from the algorithms described above. Hence, in the example, total cost reduction amounts to $43 \%$ of the reference cost, which regards the case in which the CSSD is not outsourced.

\section{Nondeterministic optimization}

In this section, we dispose of our simplifying assumption that all required nets are known precisely in advance. OT processes are unpredictable by nature for the following reasons:

1. Many of the patients arrive unexpectedly, and as emergency patients who need to be operated instantly;

2. It frequently happens that surgery of expected patients evolves in an unexpected manner, and hence that additional sterile equipment is needed;

3. Nets of sterile equipment may be incomplete, may become unsterile beforehand, et cetera;

4. Different surgeons use different nets, and the actual surgeon is not the planned surgeon.

If the variations and uncertainties regard the majority of the surgeries, and cannot be reduced, the basic logistic design to always replenish as quickly as possible to OT sterile storage may appear close to optimal. However, the larger the fraction of surgeries that can be planned, and are executed as planned, the more planning and monitoring information can be used effectively, in which case a change of logistic design may prove to reduce cost.

We start by considering some basic replenishment models, as they are currently being used in hospital practice. The two bin system is a simple and well known replenishment policy (see for instance [8]) which is also related to Just In Time systems. In a two bin system, the inventory level at the sterile storage of the OT is maximized to be two 'bins' per net. Nets are taken from bin one, until bin one is empty. Subsequently, nets are taken from bin two, until it is empty. Upon being emptied, bins are replenished so that when bin two is empty, bin one has been replenished and vice versa. Another simple policy used in practice is to always order the amount which has been used. More advanced ordering policies are for instance considered in ([3]).

Essentially, the only way to deal with unplanned usage of nets is to keep safety stock. Safety stock must not only be kept, it might also have to be replenished. The safety stock replenishment can of course be aligned with the replenishment of planned usage. We propose four different aligned replenishment policies:

1. The original planning includes only deliveries for planned usage as computed using the aforementioned dynamic programming approach. Unplanned usage for the planning period has to be delivered from designated stock. This designated stock serves as a safety stock which should last through the entire planning period. This strategy does not require any information exchange on unexpected use or replanning. 
2. The original planning includes both planned usage as well as expected demand for unplanned material usage. The optimal delivery schedule for this forecast is then computed using the dynamic programming approach described above. This approach is combined with an appropriately set initial safety stock level. Unplanned use does however not lead to changes in delivery schedule. Together with the safety stock, the current plan should suffice. As it was the case for the previous strategy, no (real time) information on usage is exchanged, and no replanning occurs.

3. Schedule only delivery for planned usage of sterile nets using the dynamic programming approach presented above, and guard against unplanned use by an initial safety stock. When the stock level drops below the safety stock level, the transportation plan is dynamically reoptimized including immediately replenishment of net types which are below safety stock level.

4. Initially the plan is to schedule delivery for planned usage and for expected demand for unplanned usage of sterile nets, using the dynamic programming approach presented above, and to guard against unplanned use by an initial safety stock. When the stock level drops below the safety stock level, the transportation plan is dynamically reoptimized including immediately replenishment of net types which are below safety stock level. In this reoptimization both planned usage and expected demand for unplanned usage are taken into account.

The first policy deals very statically with the stochastic demand. It simply arranges sufficient safety stock in advance. The second policy only uses information that is a priori known and safety stock. The third policy reoptimizes using real time information. The fourth policy uses a priori and real time information.

We compare the policies in a simulation environment. The planned demand is fixed for a week. Stochastic demand unfolds during the week. Let it be noted first though, that the nondeterministic setting is essentially different from the one discussed in the previous section with respect to differences in net types. In the previous section we argued that the types of the nets did not matter, only their volumes did. However, when keeping safety stocks, it is not the volume that counts, but also the type of the nets. Hence, the algorithms and models developed in the previous section are only valid in this nondeterministic setting if all nets are of a common type. This is for example the case when a same net is used for all types of surgery. Not a common situation in a general hospital, but a possible solution in specialized centers. The dynamic programming algorithms of the previous section can be extended to cases with multiple net types, but the state space of the dynamic programming methods grow exponentially with the number of net types [7].

The issue of composing nets will be taken into consideration after the continuation of our running example.

Example 3 In the comparison below, we say that during each morning and afternoon session of four hours, there is every hour a probability of 0.5 for unexpected changes in the OT schedule. For simplicity, we assume each unexpected usage to result in an extra use of one capacity unit of storage. Moreover, we have adapted the initial volume requirements of the example in such a way that the expected usage equals the usage implied in Example 2. Hence, the cost of 445 can serve as a lowerbound for the minimum attainable cost in this non deterministic case.

The results presented below are average results over 50 simulations. We demand for each of the policies that safety stocks are such that stock outs never occur.

\section{No expected demand planned, frozen planning}

In this scenario, a total storage space of 35 is needed, and a safety stock of 26 . The resulting total cost amounts to 638 .

2. Expected demand planned, frozen planning

In this scenario, a total storage space of 18 is needed, and a safety stock of 7 . The resulting total cost amounts to 485 .

3. No expected demand planned, replanning

In this scenario, a total storage space of 16 is needed, and a safety stock of 5 . The resulting total cost amounts to 469.

4. Expected demand planned, replanning

In this scenario, a total storage space of 14 is needed, and a safety stock of 3 . The resulting total cost amounts to 451 .

$\mathrm{Al}$ these policies in which demand is stochastic yield lower cost than the reference cost due to optimization of the sterilization logistics. In particular, we notice that the cost induced by the last scenario is very close to the cost of 445 in the optimal solution of the deterministic case.

We conclude this section by considering the issue of minimizing stock levels when taking differences between nets into account. To this purpose, we study the effect of using different net compositions under the first 
replenishment policy described above. We consider three different net compositions:

1. Each operation type has its own net, and its own stock,

2. There is one net for all operations,

3. Each operation may require multiple nets, one dedicated net, and one net which serves more than one operation type, perhaps all operation types.

Obviously, the second solution entails a lower number of nets in stock than the first solution. This may entail a decrease in storage cost. However,the number of items per net must be higher, which might cause an increase in storage cost, and in unit cost. The third solution is an unspecified combination of the first two. The example below demonstrates that he third solution allows to construct solutions with lower cost than the first two simple solutions.

Example 4 Under the same settings as described before, the safety stock levels which ensure zero stock outs are: For type A, 2 nets, for type B, 3 nets, for type C, 3 nets, for type D, 5 nets, and for type E, 5 nets. In total this amounts to 18 nets. If a common net type is used for all operation types, a safety stock level of 8 nets suffices. Hence, in this example risk pooling effects [9] lead to a reduction of the number of nets in safety stock by more than $50 \%$.

It then follows that if each type has its own safety stock, the cost can be calculated by multiplying the storage capacity cost per instrument by the number of instruments times the required stock level for each net type. For the first solution, this amounts to $9 \times((3 \times 2)+(3 \times 3)+(2 \times 3)+(2 \times 5)+$ $(2 \times 5))=9 \times 41=369$. For the second solution this amounts to $9 \times(8 \times 7)=504$. Notice that instrument unit cost is also much higher for this second solution.

A possible third solution is to put instruments $(g, h)$ into a common net, and the other instruments in separate ones. This leads to cost $9 \times((2 \times$ $2)+(2 \times 3)+(1 \times 3)+(1 \times 5)+(1 \times 5))+(2 \times 8))=$ $9 \times 34=306$. When compared to the case of a single net per operation type, the expected extra instrument usage cost for this solution can be computed as follows. Per operation, one additional (obsolete) instrument is used, and the expected number of operations equals 58. Hence, in expectation, this solution yields a cost of 364, being slightly below the original 369 (further improvement is possible by putting the components of types $g$ and $h$ in separate nets).

In the next section we consider the problem of finding an optimal net composition from a different angle.

\section{Optimizing the net composition}

In this section we explore models for optimizing the composition of the nets. This net optimization problem will be called NOP. To study the core of the problem, we focus on instrument usage cost and storage cost, and disregard transportation cost in our basic model. However, in addition to instrument related storage cost, we explicitly consider net related storage cost. This allows the model to also apply to cases in which the storage cost is partly independent of the number of instrument in the net.

In this section, we only consider the operations $A$, $B$ and $C$ from our previous example. For these operations, we need nets containing at least $(a, f, g)$ for operations of type $A$, nets containing at least $(b, f, g)$ for operations of type $B$, and nets containing at least $(c, g)$ for operations of type $C$. The minimal solution therefore uses nets $(a, f, g)$ for operations of type $A$, nets $(b, f, g)$ for operations of type $B$, and nets $(c, g)$ for operations of type $C$. It is the same as the solution presented in the previous section. This solution minimizes instrument usage cost since it never happens that nets contain instruments which are not used. However from a viewpoint of storage cost, this method is not preferable. Many nets will be required since risks are not pooled. Moreover instruments are not used as frequently as possible increasing the number of instruments needed.

The second solution presented in the previous section uses one net type for all three operations: nets containing $(a, b, c, f, g)$. This leads to an increase in instrument usage cost. The number of nets is as low as possible, yielding lower net storage cost, but instrument storage cost might still be high since even rarely used instruments are present in every net.

As mentioned before, one might expect that in between these extremes there exist better solutions, in which several operation types share a net type. For example, a net type $(a, b, f, g)$ for operation types $A, B$, and net type $(c, g)$ for operation type $C$. A further improvement is yet possible, by using more than one net per operation. For instance, a standard net $(f, g)$ can be composed, which is used for every operation type. We now give a mathematical programming formulation for a basic version of the net composition problem, and discuss its complexity. Variations and extensions can be found in [10].

The (additional) notation used to define the problem mathematically is as follows. We define a set of nets $j=1, \ldots, n$. As before, index $i=1 \ldots, m$ refers to the planning period. We use $k=1, \ldots, K$ to refer to the operations, and $l=1, \ldots, L$ to refer to the sterile 
instruments. Finally, index $t, t=1, \ldots, T$ refers to the instrument types. Basically, the problem boils down to deciding which materials go into each of of the nets, and to select nets for each of the operations such that each required material is in one of the selected nets. The model assumes that all instruments are unique. Moreover it assumes that instruments of a same type are indexed consecutively. We use the following decision variables:

- $\quad M_{j l}=1$ if net $j$ contains instrument $l$ where $j=$ $1, \ldots, n, l=1, \ldots, L, 0$ otherwise,

- $Z_{j i k}=1$ : net $j$ is used at day $i$ for operation $k$, $j=1 \ldots, n, i=1, \ldots, m, k=1, \ldots, K, 0$ otherwise item $Z_{j i k l}=1$ if instrument $l$ is contained in net $j$ and net $j$ is used for operation $k$ at day $i, 0$ otherwise.

Moreover, we introduce the following parameters:

- $\quad P_{l}$ : Instrument storage cost for instruments of type $t, t=1, \ldots, T$,

- $H:$ Net storage cost

- $S_{t}$ : Instrument usage cost for instruments of type $t, t=1, \ldots, T$

- $m_{t s}$ : The lowest index for instruments of type $t, t=$ $1, \ldots, T$,

- $m_{t e}$ : The highest index for instrument of type $t, t=$ $1, \ldots, T$,

- $N_{k t}$ : The number of instruments of type $t, t=$ $1, \ldots, T$ needed for operation $k, k=1, \ldots, K$

The problem can now be modelled using the ILP given below:

$$
\begin{aligned}
& \min \sum_{j} \sum_{t} \sum_{j} l\left(M_{j l} \times P_{t} \sum_{j} Z_{j}\right. \\
& \quad \times H \sum_{j} \sum_{i} \sum_{k} \sum_{t} \sum_{j} l Z_{j i k l} \times S_{t} \\
& \text { s.t. } \sum_{j} M_{j l} \leq 1 \quad l=1, \ldots, L, \\
& \sum_{k} Z_{j i k} \leq 1 \quad i=1, \ldots, m, j=1, \ldots, n \\
& \sum_{j} \sum_{l=m_{t s}}^{m_{t e}} Z_{j i k l} \geq N_{k t} \quad i=1, \ldots, m, k=1, \ldots, K \\
& \quad t=1, \ldots, T \\
& \quad l=1, \ldots, L
\end{aligned}
$$

$$
\begin{aligned}
Z_{j i k l} & \leq Z_{j i k} \quad j=1, \ldots, n, i=1, \ldots, m, k=1, \ldots, K, \\
l & =1, \ldots, L \\
Z_{j i k l} & \geq Z_{j i k}+M_{j l}-1, \quad j=1, \ldots, n, i=1, \ldots, m, \\
k & =1, \ldots, K, l=1, \ldots, L
\end{aligned}
$$

The first constraint models that an instrument can be in at most one net. The second constraint models that a net can only be used once per day. The remaining constraints can be interpreted as follows: Constraint (23) ensures that sufficient instruments are assigned to each operation. Constraint (24) models that an instrument $l$ from net $j$ can only be used for an operation if $M_{j l}=1$, i.e. if it is in the net. Next, we model that if an instrument $l$ of net $j$ is used for an operation $k$ at day $i$, then $Z_{j i k}=1$, i.e. the net is used for the operation. The last constraints enforces that if a net is used for an operation, and the instrument is in the net, than the instrument is seen as used, even if it is not needed. This constraint is required to appropriately model the objective function.

Now that $N O P$ is properly formulated, let us first consider its complexity.

\section{Theorem 1 NOP is strongly NP Complete.}

Proof The polynomial ILP formulation presented above entails that NOP is in NP. Its completeness follows from a reduction from the NP-Complete threedimensional matching problem (3DM). This problem is defined by Garey and Johnson [4] as followed:

Instance Three sets $A, B, C$ each containing $q$ elements, and a ternary relation $R \subseteq A \times B \times C$.

Question Does $R$ contain a subcollection $R^{\prime} \subseteq R$ such that every element of $A, B$ and $C$ occurs in exactly one member of $R^{\prime}$ ?

Notice that the cardinality of $R^{\prime}=q$ and hence that without loss of generality $\|R\| \geq q$. In the instances of $N O P$ constructed in the reduction below $\|R\|$ corresponds with $L$, the number of instruments.

We polynomially construct an instance of $N O P$ as follows. There are 3 days (i.e. $i=1,2,3$ ), and at each day $q$ operations have to be performed. The operations of day one are called $\alpha_{1} \ldots \alpha_{q}$, the operations of day two are called $\beta_{1} \ldots \beta_{q}$ and the operations of day three are called $\gamma_{1} \ldots \gamma_{q}$.

To determine which instruments are needed for every operation the following procedure will be applied. Each of the $L$ instruments, is of a unique type, and initially all operations need all $L$ instruments. For 
each $r \in R$ if $\alpha_{l} \in r$, then instrument $r$ is not needed for operation $l$ at day one, if $\beta_{l} \in r$ than instrument $r$ is not needed for operation $l$ at day two and if $\gamma_{l} \in r$ then instrument $r$ is not needed for operation $l$ at day three. The net storage cost is $L^{2}$ and instrument storage cost is 1 .

Claim The answer to $I$ is yes if and only if $I^{\prime}$ has a solution with value $q \times L^{2}+q \times(L-1)$.

Let $I$ be a yes-instance and let $R^{\prime}$ be the threedimensional matching for I. Now, for every $r \equiv$ $\left(\alpha_{u}, \beta_{v}, \gamma_{w}\right) \in R^{\prime}$, the same net is used for operation $u$ at day 1, for operation $v$ at day 2 and for operation $w$ at day 3. By definition, none of these operations need instrument $r$, and together they need all other instruments. Therefore the cost of the net consists of $L^{2}$ for net holding cost, plus $L-1$ for the instrument holding cost of the instruments contained in it. Because there are $q$ operations every day there have to be a total of $q$ nets and the total cost is $q \times m^{2}+q \times(m-1)$, completing the first part of the proof.

Now suppose $I^{\prime}$ has a solution $S^{\prime}$ of value $q \times L^{2}+$ $q \times(L-1)$. Using that $q \leq L$, we derive that this solution cannot use more than $q$ nets because the cost would be at least

$(q+1) \times L^{2}>q \times L^{2}+q \times(L-1)$

Any feasible solution uses at least $q$ nets, since there are $q$ operations each day, which yields that the solution uses exactly q nets.

Thus each net is used every day, and for an arbitrary net, the three operations for which it is used will be called $u^{\prime}, v^{\prime}$ and $w^{\prime}$. By construction, the net needs to contain $L-1$ instruments if $\left(\alpha_{u^{\prime}}, \beta_{v^{\prime}}, \gamma_{w^{\prime}}\right) \in R$, and $L$ instruments otherwise. If $S^{\prime}$ has value $q \times L^{2}+q \times(L-1)$, then it must consist of $q$ sets of $L-1$ instruments. But, this implies that every net is used for a triple of operations $\left(u^{\prime}, v^{\prime}, w^{\prime}\right)$ corresponding to a triple $\left(\alpha_{u^{\prime}}, \beta_{v^{\prime}}, \gamma_{w^{\prime}}\right) \in$ $R$. Since, the nets cover all $q$ operations, of each of the three days, the corresponding triples form a solution for $I$.

Computational experiments to solve instances of NOP by solving ILP formulations using Cplex indicate that it is very hard to solve to optimality.

\section{Concluding remarks}

Redesigning the sterile logistics processes can increase effectiveness of hospitals, as it is widely sought. The re- sults and examples in this paper indicate that significant cost reductions are possible when making appropriate use of logistic principles, operations research methods, and IT (such as RFID).

The Maasland Ziekenhuis is in the process of implementing the ideas described in this paper (among others), in its transition to a newly build customer centered hospital in 2008. The entire implementation process will take from 2005 to at least 2009, since it involves many organizational, technological and physical changes. Clever standardization of net composition, as discussed in Sections 4 and 5, and introducing pull logistics for a limited set of surgery types, is reported to have already freed over 500,000 euro in working capital, and to have lead to a comparable amount in annual savings in operational costs. Moreover, it is anticipated that OT storage space will be more than halved due to pull logistics and optimizing net composition. Extrapolating these number for the whole of The Netherlands yields a savings potential of well over 100 million euros.

We hope that the practical potential, as well the theoretical work in this paper, encourage future work on the optimization of sterile logistics. The urgency to control the cost of health care, and indeed the opportunity costs in terms of health care which currently is not provided by lack of budget, more than justifies practical and theoretical advances in this relatively unexplored area.

\section{References}

1. Bakker P (2003) Sneller Beter (in Dutch). Report, Dutch Ministry of Health

2. Carter M (2002) Diagnosis: mismanagement of resources. OR/MS Today 29(2)

3. Fineman SJ, Kapadia AS (1978) An analysis of the logistics of supplying and processing sterilized items in hospitals. Comput Oper Res 5:47-54

4. Garey MR, Johnson DS (1979) Computers \& intractability. Freeman

5. van de Klundert JJ (2005) Hoogervorst's optimaliseringsprobleem (in Dutch). StatOR 1-2:8-12

6. van de Klundert JJ, Van Merode GG, van Mulken IMT, Tummers G (2003) Het logistiek systeem van CSA en OK, een simulatiestudie (in Dutch). Maastricht University

7. Muls P (2004) Materiaalstromen in een ziekenhuis: een simulatiestudie (in Dutch). Master's thesis, Maastricht University

8. Silver E, Pyke D, Peterson R (1998) Inventory management and production planning and scheduling. Wiley

9. Simchi-Levi D, Kaminsky P, Simchi-Levi E (2000) Designing \& managing the supply chain. McGraw-Hill

10. Schadd M (2005) The operation set problem. Bachelor Thesis, Maastricht University 